removed the two sutures from the external wound. His oje felt free. On the fourth day I remored the two sutures from the conjunctiva, and found the flap adherent in its new place, and its vitality unimpaired.

The eye gradually became stronger, but I did not divide the root of the flap until ten days after the operation. The little portion of skin was very vascular and sensitive; but after the division of the root I trimmed the remaining portion within the eye without any sign of pain from the patient. It was necessary to insert a little stitch in the lid at the point of division of the flap. He went home (to Wales) with full power of moving the eye freely in every direction, but with the sight much the same as before the operation.

After a few weeks I saw the patient again. Ils eye was still free in its movement, and his cornea had cleared so much that he was able to see to work, and the eye was in every respect comfortable. The skin of the little flap remained as skin, and had not become converted into mucous membrane, as I thought would have been the case.

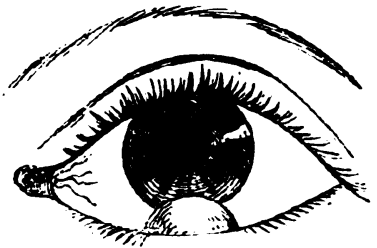

Fic. 3 . - The flap as it cxisted some weeks after the operation.

The cicatrix in the lid, as is the case when a linear scar in the proper direction has been made, was invisible, and the patient went home very grateful for his restored sight and comfort.

Case vil. Cure of Symblepharon by Operation. This case so exactly resembles that last described, that I need not narrate it fully. The patient, also an iron worker, lost both eyes by burns-the right thirteen years, and the left two years before he applied to me for advice. The right eje was gone; in the left there was symblepharon, the scar from the lower lid encroaching upon the inner part of the cornea. The same operation was performed, and with the like success; for it resulted in great improvement in the sight, and a restoration of the power of moving the eye. The skin, which had been transplanted into the eye, projected in each case as far as the edge of the lid, but entirely prevented the return of the cicatrix and the contraction, and before the operation the scar in each bad been twice divided, and had grown again to its original state, so as almost entirely to limit the movements of the eyes.

\section{OBSERVATIONS ON THE ACTION AND SOUNDS OF THE HEART.}

By R. Hrtchissox Poweld, M.D.Lond., Physician to the Royal Infirmary for Diseases of the Chest.

[Rcal before the Harvcian Society, 1852.]

IT is an evident proof of the inherent difficulties attaching to investigations connected with the circulatory apparatus, that some of the fundamental points connected with its action remain still to be established, and form a subject for debate amongst many competent and able physiologists of the past and present age. The problem to be solved, though seemingly simple aud superficial, is truly complex and recondite, involving exact anatomical knowledge, acute hearing and perception, familiarity with physical laws, more especially those affecting the properties of solids and fluids, and several minor requiremeuts. It is to be feared, moreover, that not the least among the causes of retardation are referable to views derived from and propounded upon insufficient data, partial observation, or purely hypothetical reasoning. I shall endeavour, while aroiding collateral matters, to bring together the results arrived at by
Tell known experimentalists; striving to eliminate the points of accordance from opposing and divergent results, which, however, frequently are not irreconcileable or opposing, having in view certain circumstances as regards the interval of time separating the operations, and their respective facilities and diversity of intention.

As a trustworthy basis and point of departure, I subjoin the following deductions of Volkmann, being one of the most recent and painstaking investigators. It may be romarked, that the illustrious Harrey's doctrine - that the heart is the sole moving power-is maintained throughout.

"The moving power of the heart is equal to $4 \frac{1}{1}$ lbs. 3 drs.; being composed of that force setting the blood in motion, and that overcoming resistance throughout the circulating system. There exists a direct proportion between the pressure and the velocity of the current, just as in the motion of fluids through rigid channels.

"The amount of blood ejected from the heart at each contraction is $6.2 \mathrm{oz}$., deduced from the definite relation observed to exist between the velocity of the blood and the total weight of the body. The total amount of blood in the adult being $30 \mathrm{lbs}$., the weight of blood given out in one systole being $6.2 \mathrm{oz}$., and the duration of the pulse 0.85 seconds, the period of the circuit is estimated to be 67.5 seconds." [Other observers state it to be from 25 to 30 seconds; moreover, acceleration of the pulse does not quicken the velocity of the blood-current.]

"Certain fluids either diminish or increase the circulation of the blood, alike in artificial channels, in the dead or living organism; alcohol or serum producing the former, solution of nitrate of potash the latter, effect. [The connexion of this law with the concomitant of chlorosis is obrious.]

"The special influence of the capillaries is to modify the heart's impulse into an uniform current.

"The suction power of the heart is very insignificant, the right auricle being nearly passive. The accelerating effect of inspiration being equal to its retarding, the result is null.

"The distribution of the blood is governed by other forces than the beart's action (which is essentially that of enabling the blood to pass from the origin to the termination of the blood circuit, against opposing forces); a change in the elasticity of the ressels, as also the influence of respiration, being the elements chiefly involved in this distribution.

"An equal quantity of blood is received into the heart during dilatation as leaves it during contraction; the blcodwave being diffused from ventricle to its corresponding auricle during one pulsation."

The received explanations of cardiac phenomena, with which members are presumed to be familiar, will be referred to only as agreeing or contrasting with more recent views. Volkmann concludes from his experiments that " the first sound of the heart is mainly duc to distension of the auriculo-ventricular valves, the second sound being exclusively caused by distension of the semilunar valves". This opinion was held by the late M. Bouillaud, Dr. Hope, and very closely agrees with chat of Dr. Billing. Professor Kiwisch propounds the following doctrines, as resulting from his experiments and deductions, derived from the laws of acoustics, the data furnished by auscultation, the result of vivisections, various acoustic experiments with elastic membranes, with vessels and hearts taken from the dead body. "Cardiac sounds are produced in the healthy organism cnly through the movements of its valves. Muscular sounds are acoustic delusions, arising from the vibration of air in the stethoscope and meatus, which, if obviated, no sound is heard, as shown over a contracting uterus. In the same way, the heart's stroke appears to cause, but does not in fact produce, sound." Dr. Williams's experiments (destruction of the cardiac valves, and removal of the ribs) disposes of the assertion that the heart's apex, when (said to be) tilted forward during contraction, induces sound. The apex, moreover, was shown by Dr. Corrigan not to adrance at all during contraction. But how recon- 
cile the production and existence of sound with the negation and overthrow of the valvular play?

This query will be best met after reference to the other closely connected cardiac phenomena. Let us see what has been effected by observing the kind and order of the heart's movements, and inquire if they correspond with prevalent views. Dr. Robinson asserted, as the result of his observation, in a very favourable case of denuded heart, " that the dilatation of the heart was its first movement, being, moreover, an active motion, and not a passive recoil from previous contraction, and that a distinct though minute interval existed between the act of diastole and systole, which latter was properly the second in the order of the heart's movements." (American Journal of Medical Science.)

Dr. Corrigan, many years ago, (Medical Gazette, vol. vi, p. 207), considered that the auricular (slow) contraction was synchronous with the first sound and with the heart's impulse, produced by a jet of blood from the auricles into the ventricles, and coincident with the swelling of its sides, projected forward.

A very interesting case of deficient sternum, in which the heart's play was very apparent, has been lately exhibited at the Harveian Society, in which the writer conceived he could trace such an order in the occurrence or succession of the cardiac movements, as would be compatiblc with and confirm the views here put forth. He has also another case under treatment at the Royal Infirmary for Diseases of the Chest, in which formidable aortic and mitral disease exist, attended with violent action of the heart, and visible distension of the carotids. In this case he conceives he has been able to make out a distinct, though almost inappreciable, interval between the cardiac impulse and radial pulse.-February, 1856.]

Professor Skoda has made some observations on the movements of the heart, in a case of absent sternum, which have a close bearing upon the statements just made.

An infant was born without a sternum; the thoracic cavity presented in front a cleft of a form and size corresponding : this chink was merely closed by a very thin and shallow membrane. Some days after birth, the Professor made the following observations :

At each inspiration, this membranous envelope was drawn towards the spinal column, and afterwards the anterior extremities of the ribs were bent slightly inwards; on the contrary, at each expiration, this membrane was pushed in front like a bladder. Sixty or more inspirations were counted in a minute. On applying the hand, it was distinctly perceived that the heart was placed rertically ; and when the finger was carried sufficiently deep in the direction of the vertebral column, one perceived at each systole the shock of the heart directly above the insertion of the diaphragm and at each diastole, as high as the second or third rib. On applying two fingers in this space, so as that one finger, placed superiorly, perceived the systolic contraction, whilst the other finger, put underneath, felt the diastolic shock, it was found that the heart, during its systole, glided about one inch downwards. In fact, the outline of the heart was seen, upon this membranous envelope moderately stretched between the fingers, both during the systole and diastole; and after this it might be concluded that the heart's shock, between the two points indicated, was not the result of an enlargement or diminution of this organ, but arose simply from its displacement. If the envelope was not touched, it was observed, that during expiration, precisely at the systole, the heart's outlines were marked by an elevation from above downwards upon the membrane pushed forwards: during the diastole, on the contrary, a sinking from below upwards was remarked. During inspiration the heart's shape was delineated equally at these two periods upon the membranous covering pushed towards the vertebral column. The heart beat from 120 to 130 times a minute; and was moved from above downwards exactly upon the middle of the cleft when the infant lay upon its back : on the contrary, if the baby leant to either side, then the heart deviated slightly in its movements from the median line away towards that side upon which the infant roclined. The imperfections in this case arose from the incompleteness of the opening, and from the heart being covered, thus preventing close inspection or normal examination. (1850.)

Skoda's case throws much doubt on the existence of any such order in the parietal movements of the heart as that usu. ally received; and, at all events, does not negative Dr. Robinson's assertion, which, moreover, receives strength, and falls in with Dr. Corrigan's former opinion, that the first sound coincides with the auricular contraction, and corresponding ventricular dilatation (the first of the hear:'s movements); also with his explanation of the mode of production of the impulse, as well as with the order given according to his views, before referred to;-that the auricles contract first, the rentricles second, and that the long silence then succeeds. The interval said to exist between the impulse and pulse may be thus accounted for; the latter coinciding with the ventricular contraction, closely followed by, if not continuous with, the second sound.

Dr. Herbert Davies considers the following to be the order of succession and duration occurring in the heart's movements and sounds:-First sound, short silence; second sound, long silence, which is commenced and terminated in the interval of time between two successive pulses, in a period equal to one-serentieth of a minute (taking the pulsations at 70 per minute). The second sound must appear to succeed immediately upon the first, the intervening time being a fractional period of time almost inappreciable. A beat or rhythm results (according to Barth and Roger) in a threefold manner; the time occupied in the first sound being a little less than one-thirl, in the short silence and second sound being in each one-sixth, and in the long silence very little over one-third. The relative duration of the heart's systole and diastole is estimated by Volkmann at 96:100. This discrepancy is not surprising, considering the minute fractions of time involved in the inquiry.

Now, if we assume, with Dr. Robinson and others, that dilatation constitutes the first of the heart's rhythmical movements, the first sound, which then becomes synchronous with the diastole, may be referred, in its mode of production, to the sonorous vibration of the dilating ventricles, conjointly with the rush of blood into them from the auricles. It is in accordance with the relation existing between the physical forces that sound should result from a certain amount of resistance being offered to the injected blood, consequent upon arrest or retardation of motion. The almost simultaneous ventricular contraction (coinciding with the pulsation in the arteries) limits this source of sound; the second sound resulting from an extra-ventricular (valvular?) cause-possibly from the onward current of blood into the aorta.

\section{PLACENTA PREVIA.}

By Johx Aunraxn, Fsq., Tintern.

Sone cases of placenta provia having been lately reported in the Joursal, I forward one which occurred in my practice; and which, I think, is sufficiently interestins for publication.

I was called to Mrs. J., aged 45 years, a woman living about three miles from this place, between 7 and 8 A.N., July 17 th, 1854. She was pregnant with her eighth child, and expected her confinement in about a fortnight. She told me that for the two prerious days she had felt a weight and bearing down sensation in the lower part of the bowels; and that about five o'clock this morning, whilst she was making water, a large quantity of clotted blood came away, and she became faint. When I arrived, she had recovered from the faintness; the pulse was full, and the hamorrhage bad ceased. On examination, I found the os uteri, which was very high and reached with difficulty, dilated to about the size of a shilling; but I could not, for a certainty, make out the presentation. I waited for some time, but as there were no pains or hamorrhage, and I had some urgent cases 